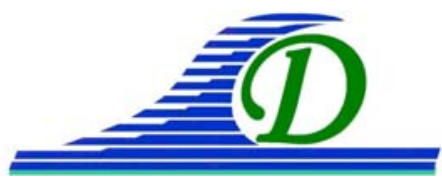

XIII İ̀es Journées Nationales Génie Côtier - Génie Civil Dunkerque, 2-4 juillet 2014

DOI:10.5150/jngcgc.2014.045 @ Editions Paralia CFL

disponible en ligne - http://www.paralia.fr - available online

\title{
Quelles perspectives face à la dynamique préoccupante des plages d'Assouindé Valtour et du Club Nautique, respectivement au Sud-Est et au Sud-Ouest de la Côte d'Ivoire
}

\section{Koffi Philibert KOFFI ${ }^{1}$, Kouadio Salomon YAO ${ }^{1}$, Jacques ABE ${ }^{1}$, Célestin HAUHOUOT ${ }^{2}$, Siaka Barthélémy BAMBA ${ }^{1}$}

\author{
1. Centre de Recherches océanologiques d'Abidjan, Côte d'Ivoire. \\ yksalomon3@yahoo.fr \\ 2. Institut de Géographie Tropicale, Université Félix Houphouët-Boigny, Abidjan, Côte \\ d'Ivoire.
}

\section{Résumé :}

Le segment de plage d'Assouindé Valtour de même que le périmètre littoral de SanPédro sont le siège de différents enjeux économiques, respectivement à l'extrême Est et Ouest du littoral ivoirien. Cependant, ces sites qui connaissent des évolutions régressives naturelles critiques du rivage sont soumis à l'impact des aménagements côtiers. Cette dynamique des rivages, en contradiction avec les perspectives de développement économique en présence, justifie l'attention accordée à l'analyse de la dynamique morpho-sédimentaire de ces espaces. A Assouindé, l'observation à court terme du paysage met en évidence la coexistence d'évènements contradictoires, traduites par une forte résilience de la plage consécutive à une importante déstabilisation du rivage. Ce processus attire davantage la convoitise des investisseurs, ignorants dans leur grande majorité de la véritable nature labile de cet espace. Cette communication se propose donc de faire le bilan du suivi morpho-dynamique à moyen et long termes (2008-2012 et 1985-2012) de ces espaces littoraux, afin de mettre à la disposition des décideurs et aménageurs des éléments de prise de décision.

Mots-clés : Dynamique sédimentaire, Erosion côtière, Assouindé, San-Pédro, Côte d'Ivoire.

\section{Introduction}

La façade maritime de Côte d'Ivoire présente, d'Ouest en Est, une diversité morpho lithologique du paysage. Cet atout a été mis à profit dans les premières décennies de l'indépendance du pays, dans le cadre de la politique de diversification des pôles et ressources économiques, partant le désenclavement des régions. On note ainsi dans le Sud-Ouest plusieurs opérations de développement dont l'épine dorsale est l’aménagement du port de San-Pédro. 


\section{Thème 2 - Dynamique sédimentaire}

Au Sud-Est, l'aspect attrayant de la zone littorale a suscité la mise en place d'un cadre législatif et réglementaire, dans les années 1970 à 1974, en vue de favoriser l'investissement privé dans le secteur du tourisme. Cette politique a facilité l’implantation de deux villages de vacances de renommée internationale à Assouindé: le Club Méditerranée et le Valtour.

Ces différents aménagements, bien que sources de développement économique, contribuent à modifier les équilibres sédimentaires côtiers, en aval dérive des infrastructures portuaires. Le périmètre de plage du Club Nautique à l'Est du port de San-Pédro ainsi que la baie de Port-Bouet à Abidjan illustrent cette évolution (HAUHOUOT, 2000 ; ABE, 2005 ; YAO et al., 2010 ; KONAN, 2012 ; YAO, 2012).

Par ailleurs, le secteur d'Assouindé Valtour, du fait de la morphologie du lido, subit également l'influence naturelle des aléas météorologiques, avec une dynamique régressive globalement faible, mais des submersions de plus en plus fréquentes ( $\mathrm{ABE}$, 2005 ; KONAN, 2012).

A l'analyse, la dynamique actuelle des périmètres littoraux des pôles économiques susmentionnés contrarie les politiques de développement pour assurer leur valorisation convenable, dans un contexte de relance économique du pays. Cet état de fait mérite que l'on se penche résolument sur les dispositions à prendre pour réduire les risques encourus. Cela passe nécessairement par une connaissance approfondie du milieu et l'analyse des caractéristiques morpho-sédimentaires des sites constitue une démarche importante. La présente note concerne l'étude de la dynamique des sites du Club Nautique et d'Assouindé Valtour. Leurs caractéristiques morphologiques intimement liées aux forçages naturels et anthropiques seront examinées sur des échelles de temps variables, afin d'évaluer leur vulnérabilité indispensable pour les perspectives de protection à envisager.

\section{Présentation des sites et méthodologie}

\subsection{Sites d'étude}

Les secteurs de plage étudiés se situent respectivement sur les périmètres littoraux des côtes de plateaux rocheuses du sud-ouest et des côtes basses sablonneuses du sud-est (voir figure 1).

Dans le premier cas, la morphologie générale de la côte est caractérisée par la disposition des segments de plage en échelon où de minces cordons littoraux rectilignes sont décalés les uns par rapport aux autres, séparés par des caps rocheux plus résistants. Ce tracé d'ensemble tient de la tectonique locale ou de l'érosion différentielle du socle précambrien (TRICART, 1957 ; HINSCHBERGER \& POMEL, 1972 ; TASTET, 1985). Le segment de plage du Club Nautique dans cette zone s'étire sur environ $1 \mathrm{~km}$ de côte à l'Est du chenal portuaire de San-Pédro, de la pointe Bato à l'Ouest à la pointe 


\section{XIII ${ }^{\text {èmes }}$ Journées Nationales Génie Côtier - Génie Civil \\ Dunkerque, 2-4 juillet 2014}

Bapo à l'Est (voir photo1), entre les latitudes $4^{\circ} 44^{\prime} 30.44^{\prime \prime}$ et $4^{\circ} 44^{\prime} 49.37^{\prime \prime}$ Nord et les longitudes 6³6'28.79" et 6³6'04.53" Ouest.

Plus à l'Est, la plage d'Assouindé Valtour, porte sur environ 0,5 Km de côte, entre les latitudes $5^{\circ} 09^{\prime 28.46^{\prime \prime}}$ et $5^{\circ} 09^{\prime} 25.95^{\prime \prime}$ Nord et les longitudes $3^{\circ} 27^{\prime} 42.61^{\prime \prime}$ et $3^{\circ} 27^{\prime} 26.20^{\prime \prime}$ Ouest (voir photo 2). Elle s'inscrit sur le mince cordon littoral entre l'extrémité Ouest de la lagune Aby et la mer qui forme une presqu'île très étroite longue de $20 \mathrm{~km}$ à vol d'oiseau, depuis Assouindé jusqu'à Assinie Mafia. C'est un cordon barrière très bas avec des plages de sable fin et blanc en pente de plus en plus douce. Ce cordon connaît aujourd'hui une urbanisation croissante avec le développement de stations balnéaires dont les célèbres villages de vacances (le Club Méditerranée et Valtur) autour desquels plusieurs établissements modestes et des résidences (luxueuses villas et paillotes) ont été construits. Cette zone constitue une destination très prisée par de nombreux touristes.

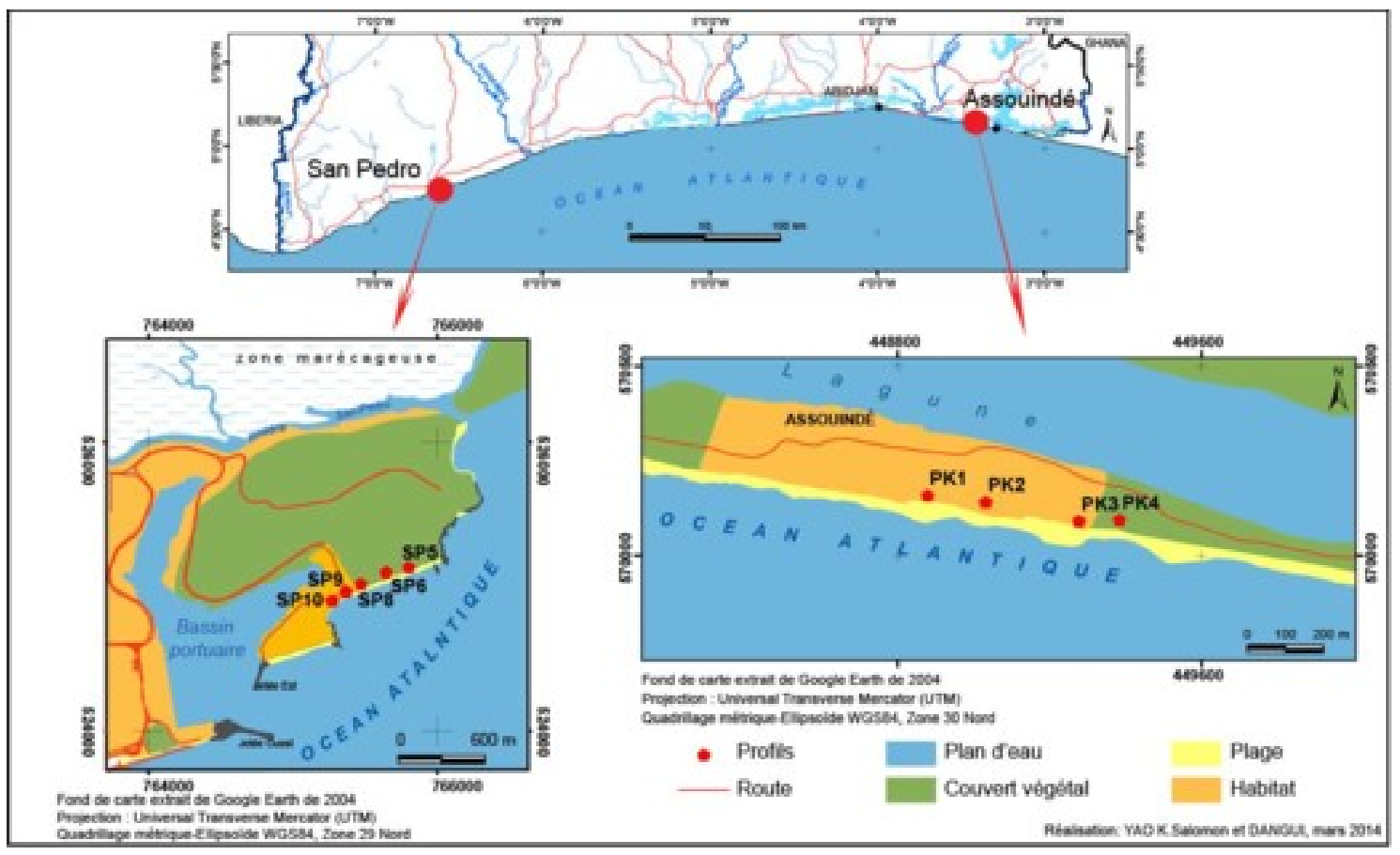

Figure 1. Zone d'étude. 


\section{Thème 2 - Dynamique sédimentaire}

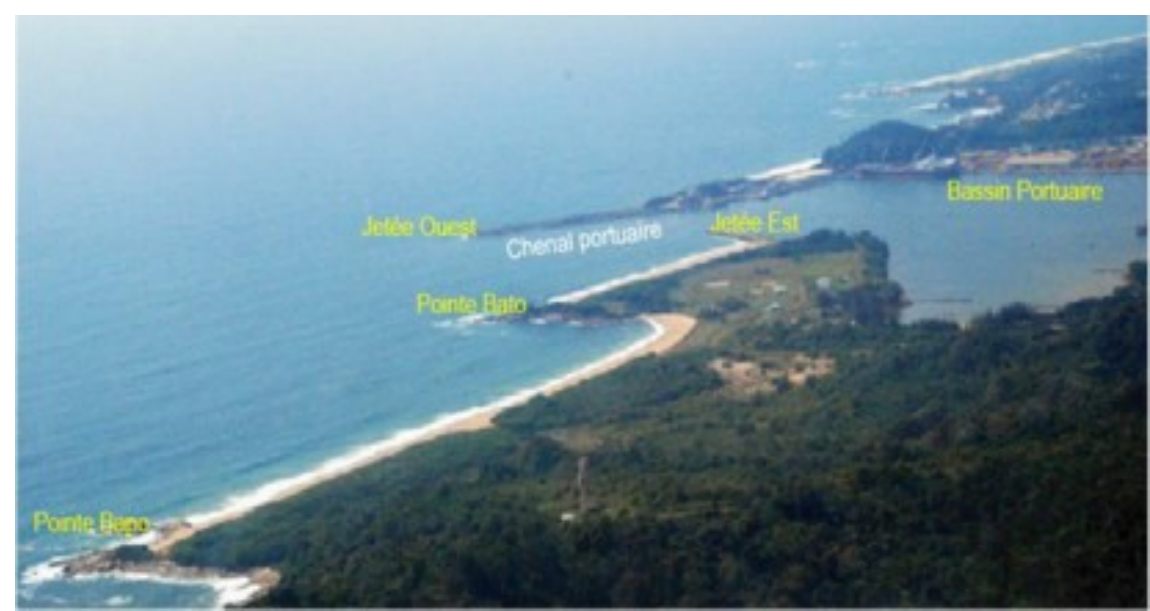

Photo 1. Segment de plage du Club Nautique (Est du chenal Portuaire de San-Pédro).

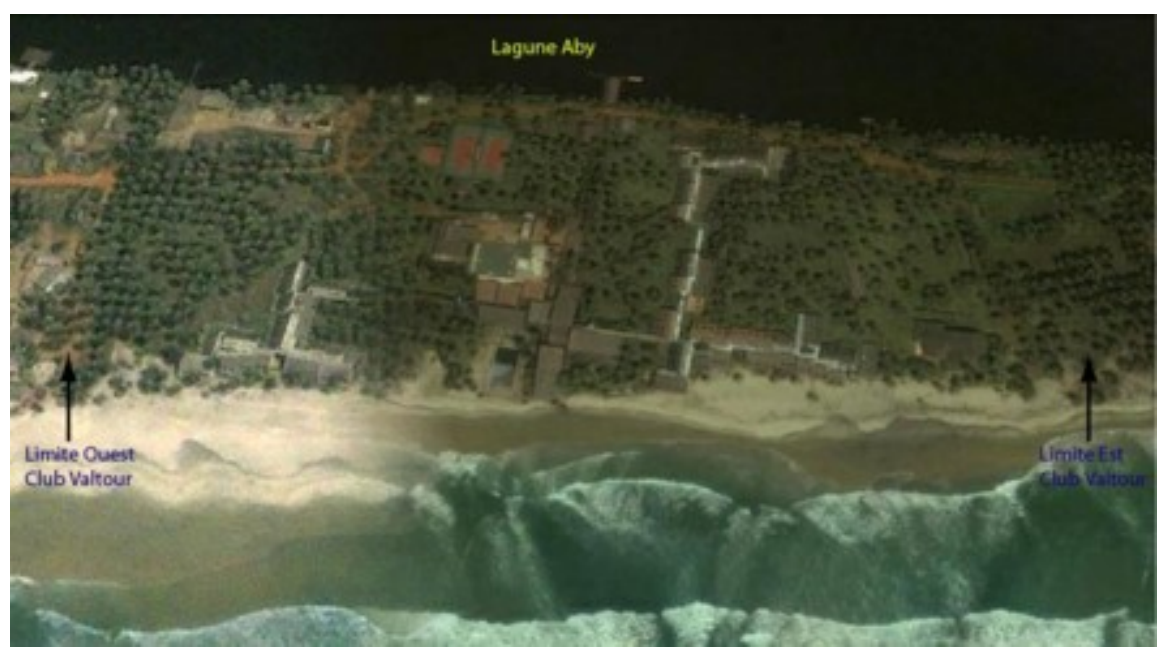

Photo 2. Segment de plage d'Assouindé Valtour.

\subsection{Méthodologie}

La dynamique des segments de côte du Club Nautique et d'Assouindé Valtour est appréhendée à partir de l'analyse des levés topographiques successifs de profils de plage. Ces levés ont été réalisés à l'aide de matériel topographique (Niveau de Chantier Leica NA730, tachéomètre Wild RDS, conformément à la méthode décrite par IBE \& QUENNELEC (1989). Ils reposent sur un réseau de 4 à 5 profils respectivement à Assouindé et à San-Pédro. L'importance des différents réseaux procède du suivi plus ou moins continu de certains profils sur une longue série (SP 10 à San-Pédro, PK1 et PK4 à Assouindé sur la période 1985/1987 - 2012). Les profils sont établis sur des repères de référence (deux bornes ou piquets au moins) implantés en arrière plage le long de la côte et espacés d'un intervalle variant entre 100 et $150 \mathrm{~m}$. Les conditions de levés topographiques des profils de plage sont idoines. Ils ont été effectués autour des marées basses de vives-eaux. En outre, ils couvrent les variations des climats de houles le long 


\section{XIII ${ }^{\text {èmes }}$ Journées Nationales Génie Côtier - Génie Civil \\ Dunkerque, 2-4 juillet 2014}

du littoral ivoirien, caractérisés par une forte agitation au cours des mois de mai à août et par une agitation faible à modérée le reste de l’année.

\section{Résultats}

3.1 Dynamique du segment de plage Club Nautique (Est Chenal portuaire) de 2008 à $\underline{2012}$

De la pointe Bato (à l'Ouest) à la pointe Bapo (à l'Est) le segment de côte Club Nautique présente des microfalaises littorales de moins en moins importantes dont l'évolution tient des climats de houles au cours de l'année. L’analyse des levés topographiques montrent dans l'ensemble une variation saisonnière du modélé de la plage, traduite par une alternance de profils érodés (période d'agitation) et de profils accrétés (période de beau temps). Sous l'action du déferlement perpétuel des vagues sur les flancs des microfalaises (pendant la période d'agitation) on assiste à une déstabilisation de la microfalaise entaillé d'un talus de 2 à 3 m environ dans le sable argileux de l'arrière plage. Ce segment de côte enregistre donc une évolution régressive qui se résout quasiment à celle observée au cours des périodes d'agitation de 2008 à 2009 puis de 2010 à 2012. De 2009 à 2010, on note un faible recul de la ligne de rivage voire une stabilité (voir figure 2). La tendance globale de la dynamique de ce site entre 2008 et 2012 est à l'érosion. La vitesse de recul est de l'ordre de 1m/an. Ce repli de la ligne de rivage, non uniforme le long du segment de côte, apparaît plus accentué ( $\geq$ $1 \mathrm{~m} / \mathrm{an}$ ) à proximité de la pointe Bato (voir figure 3). Elle diminue en direction du cap à l'extrême Est ( $<1 \mathrm{~m} / \mathrm{an}$ ) avec des points quasi stables dans la zone centrale. L'évolution régressive rapide du rivage vers la pointe Bato menace de disparition la borne repère avant situé à $7 \mathrm{~m}$ du rivage en 2008 (voir photo 3).

\subsection{Dynamique du segment de plage Club Nautique de1985 à 2012}

Sur le long terme, les variations régulières des bornes repère arrière selon la période d'observation ne permettent pas d'apprécier de façon efficiente l'évolution du site. Elle est retracée à partir de l'analyse de la dynamique du profil SP10 correspondant au profil P13 ayant traversé le temps de 1985 à 2012. Ainsi, la période 1985-1986 enregistre une dynamique remarquable avec un taux moyen de recul du rivage de l'ordre de $4 \mathrm{~m} / \mathrm{an}$. Ce fort taux de recul est à mettre en relation avec la tempête survenue en mai 1986. De 1993 à 1997, la vitesse moyenne de recul du rivage est estimée à $1 \mathrm{~m} / \mathrm{an}$. Cette tendance est celle observée sur la période récente de 2008 à 2012 (voir figure 4). 
SP 05

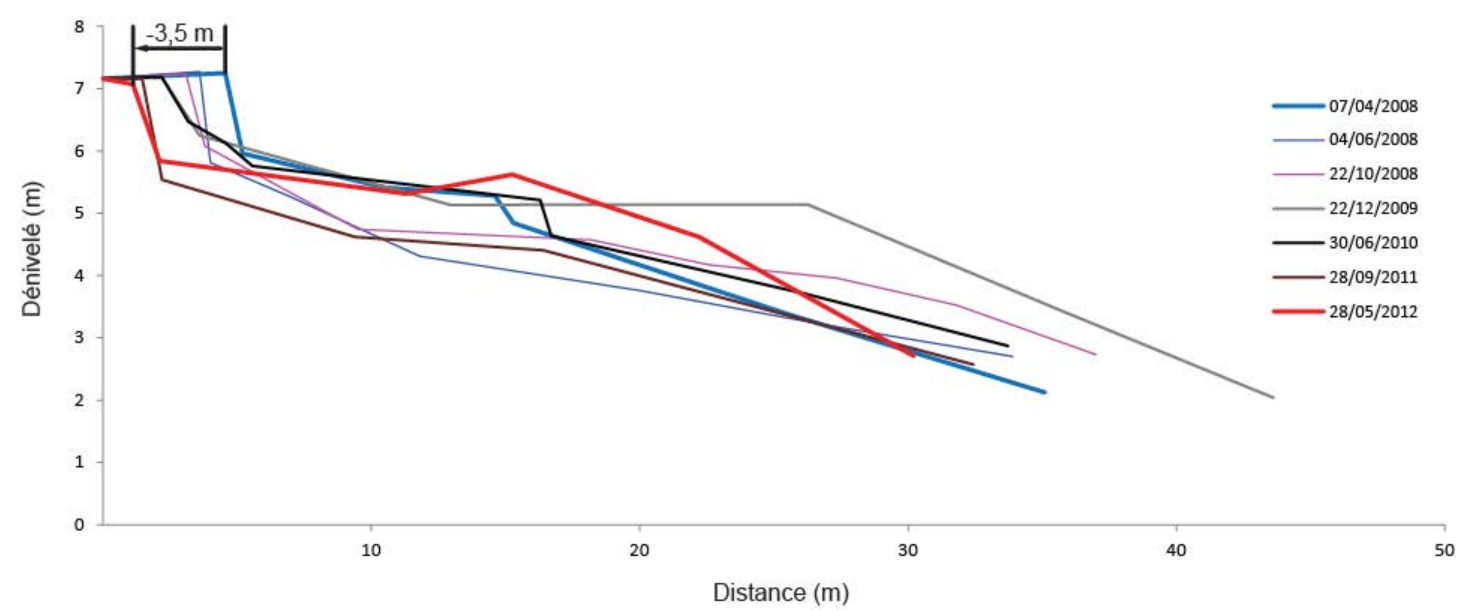

Figure 2. Evolution morphologique et tendance évolutive du rivage au droit du profil SP 05 entre 2008 et 2012.

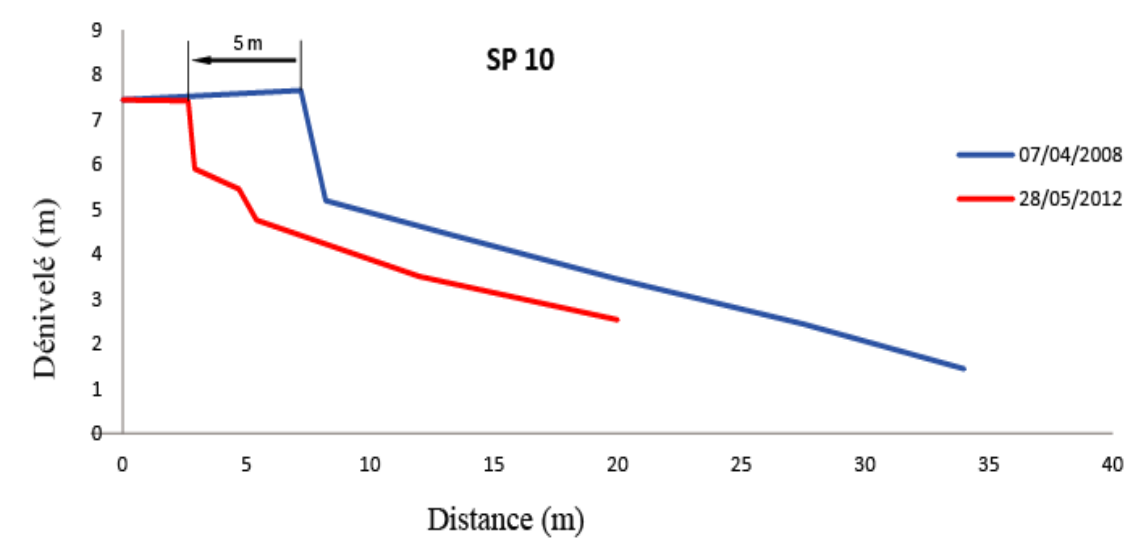

Figure 3. Tendance évolutive du rivage au droit du profil PK 10 entre 2008 et 2012.

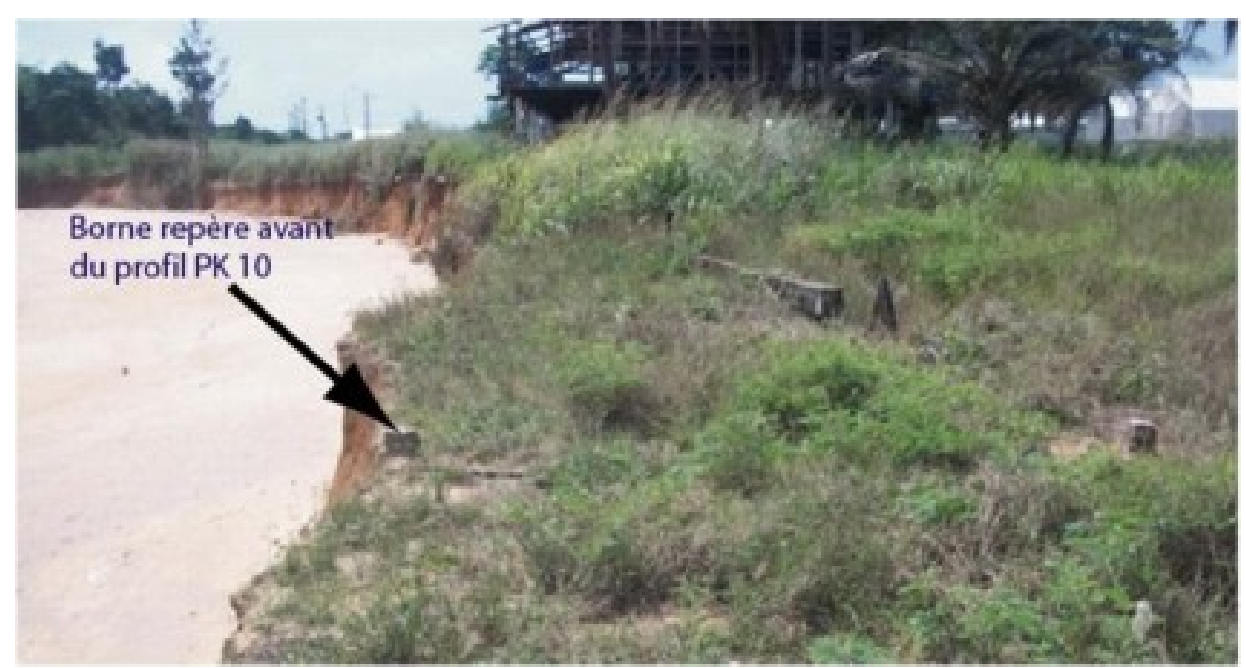

Photo 3. Borne repère avant du profil PK 10 à même le rivage (décembre 2013). 


\section{XIII ${ }^{\text {èmes }}$ Journées Nationales Génie Côtier - Génie Civil \\ Dunkerque, 2-4 juillet 2014}

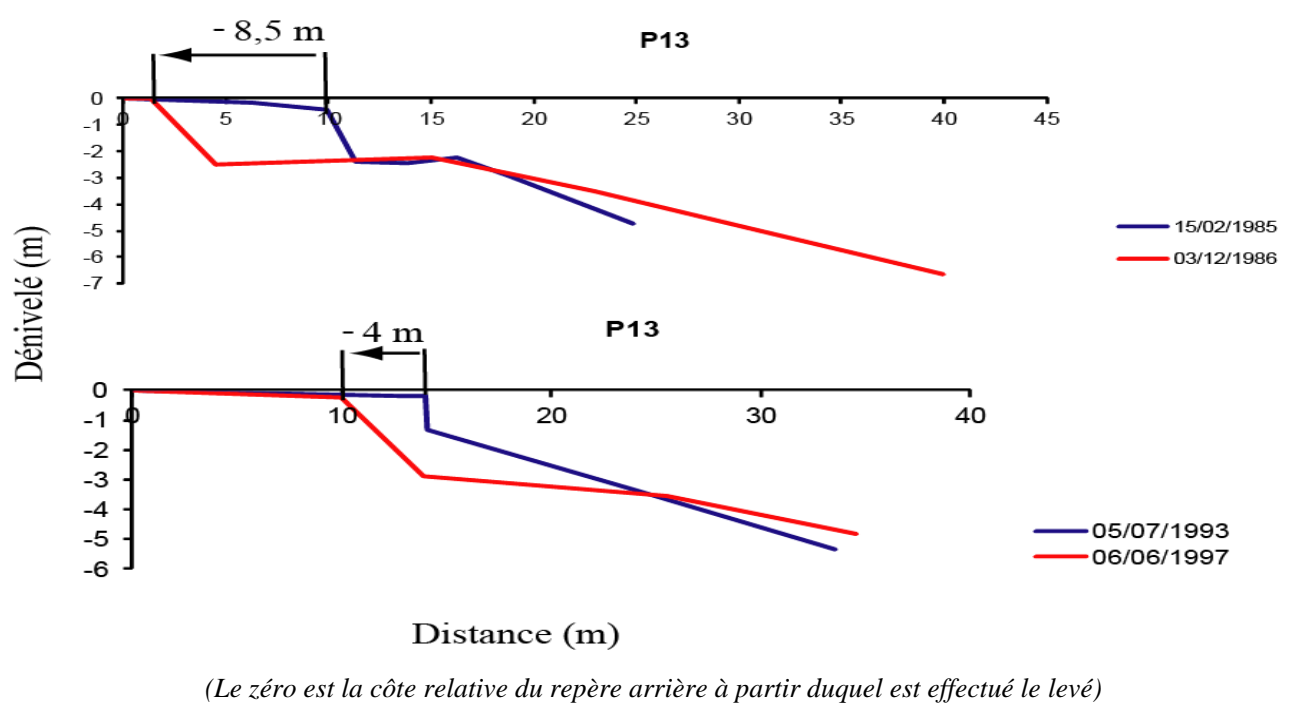

Figure 4. Tendance évolutive du rivage au droit du profil P13 entre 1985 et 1997.

3.3 Dynamique du segment de plage Assouindé Valtour de 2008 à 2012

A l'instar des profils du sud-ouest, les profils sur le cordon littoral d'Assouindé à l'extrême sud-est, présentent également une alternance de mouvements sédimentaires qui est le reflet de la variation du climat de houle sur le littoral ivoirien (voir figure 5). L'érosion du bas estran observée de mai à début octobre 2008 est relayée par un engraissement spectaculaire d'octobre 2008 à mars 2009 au niveau du profil PK1. Cette dynamique précède un important démaigrissement et recul du rivage d'environ $3 \mathrm{~m}$, de mars à juillet 2009. La forte évolution régressive de la plage est le fait de l'agitation enregistrée au cours du mois de juillet 2009. De juillet à décembre 2009, l'estran se reconstitue quasiment. Les jets de rives successifs induisent une progradation du rivage de l'ordre de $3 \mathrm{~m}$, en dépit des fluctuations des mouvements sédimentaires au niveau des mi et bas estrans. La tendance évolutive globale au droit du profil PK1 est à la stabilité du rivage avec un démaigrissement non négligeable de l'estran entre 2008 et 2012.

Plus à l'Est, la plage subit dans le même temps une fluctuation des mouvements sédimentaires de mai 2008 à décembre 2009 avec une progradation du rivage de l'ordre de $5 \mathrm{~m}$. En revanche, de décembre 2009 à octobre 2012, la dynamique est au recul du trait de côte et à l'érosion de l'estran. Dans l'ensemble, on assiste à une migration de la crête du cordon à l'intérieur des terres sur environ 11 m, de 2008 à 2012. L'évolution des profils sur le court terme traduit dans cette zone une capacité de résilience naturelle de la plage.

\subsection{Dynamique du segment de plage Assouindé Valtour de 1985 à 2012}

L'analyse de la tendance évolutive du rivage sur le long terme révèle un recul du trait de côte de l'ordre de 16 à 17 m entre 1985/1987 et 2012 respectivement au droit des profils 
PK1 et PK4 (voir figure 6). Soit une vitesse de recul de l'ordre de 0,6 m/an. Cette tendance évolutive du rivage se superpose au démaigrissement de la plage et à l'affaissement progressif de la crête du cordon qui l'expose à une forte accessibilité des vagues.

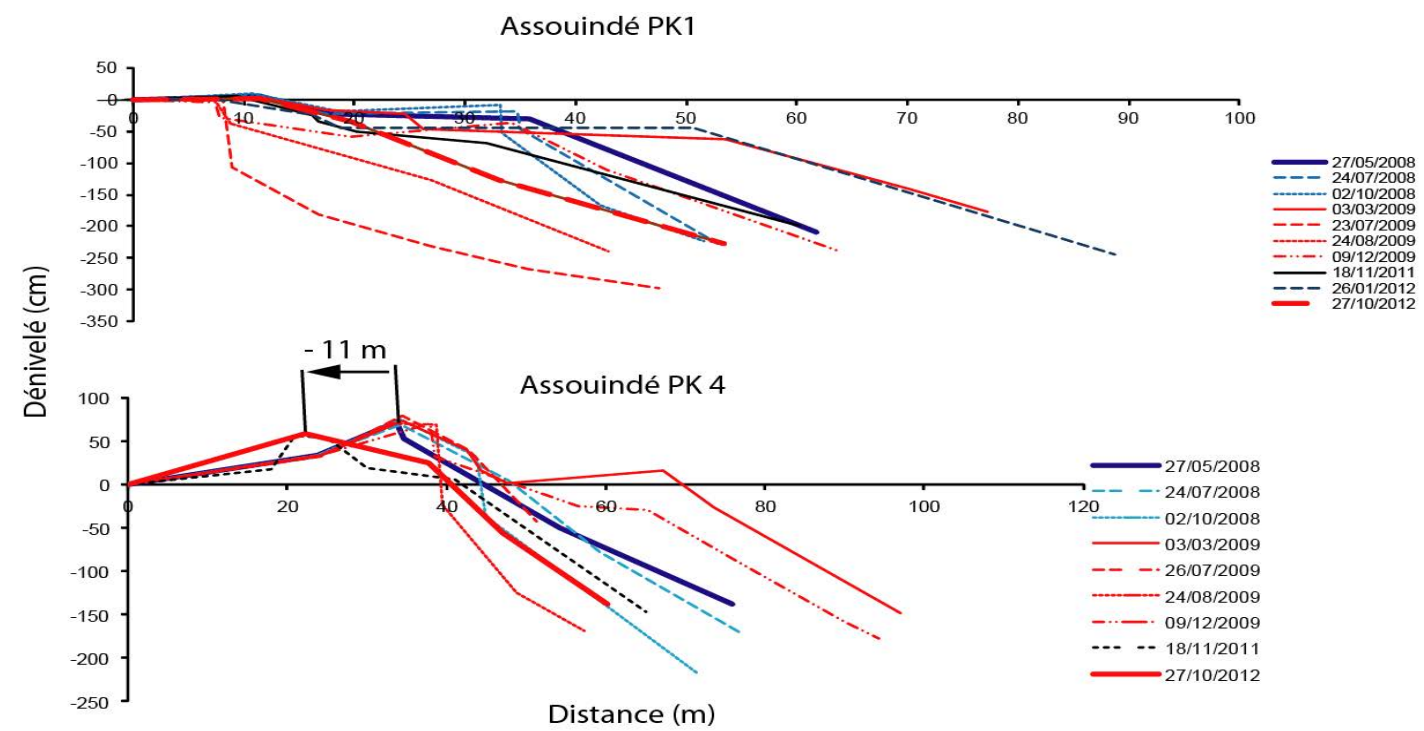

(Le zéro est la côte relative du repère arrière à partir duquel est effectué le levé)

Figure 5. Evolution morphologique et tendance évolutive du rivage au droit des profils PK01 et PK04 entre 2008 et 2012.

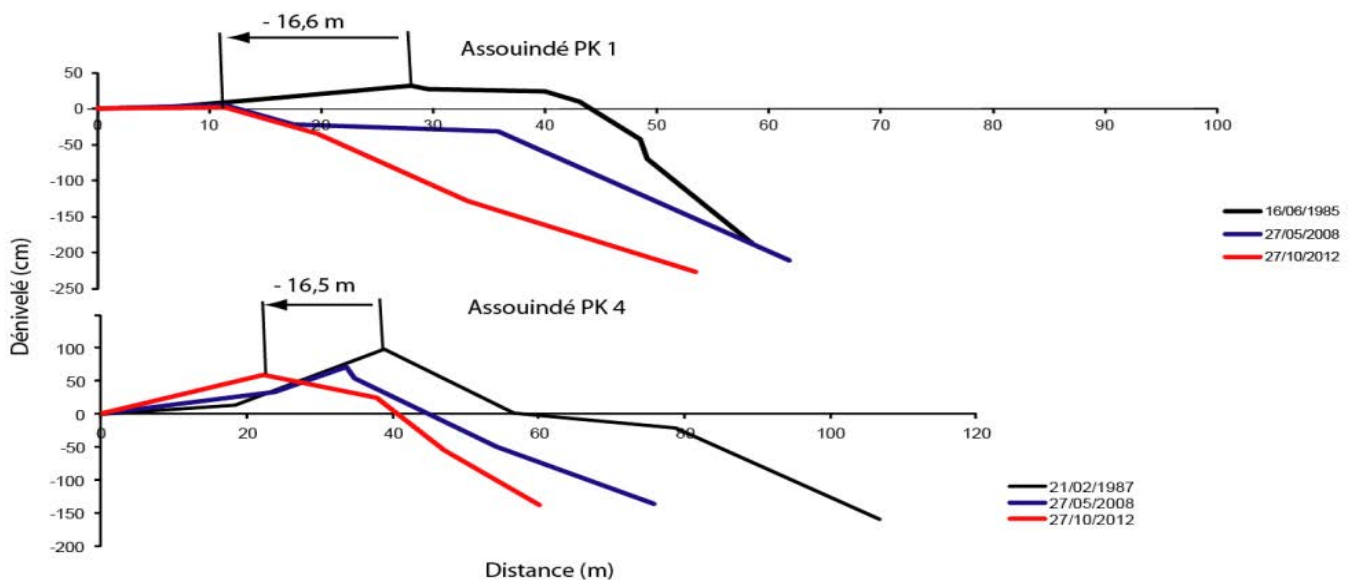

(Le zéro est la côte relative du repère arrière à partir duquel est effectué le levé)

Figure 6. Tendance évolutive du rivage au droit des profils PK01 et PK04 entre 1985/1987 et 2012. 


\section{XIII ${ }^{\text {èmes }}$ Journées Nationales Génie Côtier - Génie Civil \\ Dunkerque, 2-4 juillet 2014}

\section{Discussion}

L'analyse de la dynamique des sites Club Nautique et Assouindé Valtour, met en exergue une évolution régressive variable selon les sites. Les observations en continue ont montré que le fonctionnement des milieux littoraux, est fondamentalement nonlinéaire dans le temps et dans l'espace.

Bien que les réponses morphologiques soient le reflet des forçages océaniques, la dynamique à court et à long terme à l'Est du port de San-Pédro indique des vitesses de recul de l'ordre de $1 \mathrm{~m} / \mathrm{an}$ qui sont en général renforcées par les aménagements portuaires et les évènements exceptionnels de type tempête. Ces résultats corroborent les travaux d'HAUHOUOT (2000), ABE (2005), YAO et al. (2010) et YAO (2012). Cette tendance évolutive du site contraste avec les plans d'aménagement (parc à conteneurs, dépôt pétrolier) sur le cordon littoral séparant la mer de la darse Est, prévus dans les projets de modernisation du Port Autonome de San-Pédro.

A Assouindé Valtour, la capacité de résilience du système qui permet naturellement un retour à l'équilibre sur le court terme, indique une stabilité précaire, soulignée par $\mathrm{ABE}$ (2005) et KONAN (2012).

Cet équilibre de la plage ne doit donc pas faire perdre de vue la fragilité de cette zone lorsqu'elle est observée sur le long terme. Les vitesses de recul enregistrées sont certes faibles, de l'ordre de $0,5 \mathrm{~m} / \mathrm{an}$, mais incompatibles avec la configuration morphologique du site. L'étroitesse du cordon et l'abaissement progressif de sa crête l'expose à de fréquentes submersions pendant les périodes de fortes agitations. Cet état de fait pourrait constituer un frein à l'investissement dans cette zone. Or, le secteur touristique devrait contribuer à hauteur de $2 \%$ du Produit Intérieur Brut (PIB) d'ici à 2015, dans la perspective de la relance économique actuelle du pays (source APA, 20 novembre 2013).

\section{Conclusion}

L'étude de la dynamique des segments de plage Club Nautique (San-Pédro) et d'Assouindé valtour (Assinie) indique une tendance évolutive à l'érosion sur le long terme (1985-2012), avec les vitesses respectives de recul du rivage de 1 et 0,5 m/an. A cours terme (2008-2012), la dynamique régressive de la côte apparaît certes plus spectaculaire à San-Pédro qu'Assouindé où les plages enregistrent une forte capacité de reconstitution. Mais ces deux secteurs présentent des grandeurs de vulnérabilité du même ordre du fait des enjeux économiques en présence. Aujourd’hui, la menace des projets d'aménagements sur ces périmètres littoraux par les risques naturels est réelle et permanente dans une perspective d'une élévation du niveau de la mer au cours du siècle prochain. Des réactions au coup par coup pour tenter de porter remède à des dégradations peuvent être irréversibles. Les mesures correctives à envisager doivent donc tenir compte de cette réalité. Le choix de l'option sera fait en s'appuyant sur les meilleures connaissances du milieu, notamment les études sur modèle sédimentologique 
comme outil de prédiction de l'évolution de la morphodynamique et du trait de côte, tout ceci couplé à une analyse coût/bénéfice.

\section{Références bibliographiques.}

ABE J. (2005). Contribution à la connaissance de la morphologie et de la dynamique du littoral ivoirien (cas du littoral d'Abidjan) Essais de modélisation en vue d'une gestion rationnelle. Thèse, Université de Cocody-Abidjan, 345 p.

HAUHOUOT C. (2000). Analyse et cartographie de la dynamique du littoral et des risques naturels côtiers en Côte d'Ivoire. Thèse, Université de Nantes, 289 p.

HINSCHBERGER F., POMEL R. (1972). La morphologie des côtes rocheuses entre Monogaga et Sassandra (Côte d'Tvoire). Ann. Univ. Abidjan, série G (Géographie), tome IV, pp 7-37.

IBE A.C., QUELENNEC R.E. (1989). Methodology for assessment and control of coastal erosion in West and Central Africa. UNEP Regional seas Report and studies, $107 \mathrm{p}$.

KONAN E. (2012). Etude de caractérisation morpho-dynamique du cordon littoral sableux ivoirien à l'est d'Abidjan avant et après la tempête exceptionnelle d'août 2007. Thèse de Doctorat Unique. Univ. Felix Houphouët Boigny 187 p.

TASTET J.P. (1985). Le littoral ivoirien : géologie, morphologie, dynamique. Ann. Univ. Abidjan, Série c, T. XXI-B, pp 189-218.

TRICART J. (1957). Aspect et problèmes géomorphologiques du littoral occidental de Côte d'Ivoire. Bull. IFAN, série A (XIX), pp 1-20.

YAO K.S. (2012). Etude de la dynamique sédimentaire du littoral occidental ivoirien entre Tabou et Sassandra: Approches morpho-bathymétriques, sédimentologiques et exoscopiques. Thèse de doctorat unique, université Félix Houphouët Boigny de Cocody-Abidjan, 197 p.

YAO K.S., ABE J., BAMBA S.B., KONAN K.E., AKA K. (2010). Dynamique d'un périmètre littoral portuaire : la côte de San-Pédro, Sud-Ouest de la Côte d'Ivoire. Revue Paralia, Vol. 3, pp 2.1-2.12. http://dx.doi.org/10.5150/revue-paralia.2010.002 